\title{
Reversal-Bounded Counter Machines Revisited ${ }^{\star}$
}

\author{
Alain Finkel ${ }^{1}$ and Arnaud Sangnier ${ }^{1,2}$ \\ ${ }^{1}$ LSV, ENS Cachan, CNRS \& ${ }^{2}$ EDF R\&D \\ 61 av. du pdt Wilson 94230 Cachan. France \\ \{finkel, sangnier\}@lsv.ens-cachan.fr
}

\begin{abstract}
We extend the class of reversal-bounded counter machines by authorizing a finite number of alternations between increasing and decreasing mode over a given bound. We prove that extended reversal-bounded counter machines also have effective semi-linear reachability sets. We also prove that the property of being reversal-bounded is undecidable in general even when we fix the bound, whereas this problem becomes decidable when considering Vector Addition System with States.
\end{abstract}

\section{Introduction}

The verification of infinite state systems has shown in the last years to be an efficient technique to model and verify computer systems. Various models of infinite-state systems have also been proposed as for instance counter systems, lossy channel systems, pushdown automata, timed automata, etc, in order to obtain an automatic verification procedure. Among them, counter systems which consist in finite automata extended with operations on integer variables enjoy a central position for both theoretical results and maturity of tools like FAST [3], LASH [16] and TREX [1].

Reachability problem for counter systems. It has been proved in [20] that Minsky machines, which correspond to counter systems where each counter can be incremented, decremented or tested to zero, have an undecidable reachability problem, even when they manipulate only two counter variables. Because of that, different restrictions over counter systems have been proposed in order to obtain the decidability. For instance, Vector Addition Systems with States (or Petri nets) are a special class of counter systems, in which it is not possible to perform equality tests (equivalent to zero-tests), and for which the reachability problem is decidable [14,19].

Counter systems with semi-linear reachability sets. In many verification problems, it is convenient not only to have an algorithm for the reachability problem, but also to be able to compute effectively the reachability set. In the past, many classes of counter systems with a semi-linear reachability set have been found. Among the VASS (or Petri nets), we distinguish the BPP-nets [5], the cyclic Petri nets [2], the persistent Petri nets [15,18], the regular Petri nets [21], the 2-dimensional VASS [9]. In [10], the class of reversal-bounded counter machines is introduced as follows : each counter can only perform a bounded number of alternations between increasing and decreasing mode. The author shows that reversal-bounded counter machines have a semi-linear reachability set and these results have been extended in [11] authorizing more complex guards and

\footnotetext{
* Partly supported by project AVERISS (ANR-06-SETIN-001)
} 
restricting the way the alternations are counted. In [17], it has been shown that most of the counter systems with a semi-linear reachability set are in fact flattable, which means that their control graph can be replaced equivalently w.r.t. reachability, by another one with no nested loops. In fact, it has been proved in [6], that counter machines with no nested loops in their control structure have a semi-linear reachability set.

Our contribution. In this paper, we first propose an extension of the definition of reversal-bounded machines saying that a counter machine is $k$-reversal-b-bounded if each counter does at most $k$ alternations between increasing and decreasing mode above a given bound $b$. We show that these new reversal-bounded counter machines do also have a semilinear reachability set, which can be effectively computed. We study the decidability of the reversal-boundedness of a given counter machine, proving that the only case, which is decidable, is the one when the two parameters $b$ and $k$ are provided. Finally, we study reversal-bounded VASS, showing that one can decide using the coverability graph whether a VASS is reversal-bounded or not. Doing so, we propose a new recursive class of VASS with semi-linear reachability sets which contains all the bounded VASS. Furthermore, to the best of our knowledge, it is not known whether one can or cannot decide if a VASS has a semi-linear reachability set or if it is flattable.

Due to lack of space, some details are omitted and can be found in [7].

\section{Preliminaries}

\subsection{Useful notions}

Let $\mathbb{N}$ (resp. $\mathbb{Z}$ ) denotes the set of nonnegative integers (resp. integers). The usual total order over $\mathbb{Z}$ is written $\leq$. By $\mathbb{N}_{\omega}$, we denote the set $\mathbb{N} \cup\{\omega\}$ where $\omega$ is a new symbol such that $\omega \notin \mathbb{N}$ and for all $k \in \mathbb{N}_{\omega}, k \leq \omega$. We extend the binary operation + and to $\mathbb{N}_{\omega}$ as follows : for all $k \in \mathbb{N}, k+\omega=\omega$ and $\omega-k=\omega$. For $k, l \in \mathbb{N}_{\omega}$ with $k \leq l$, we write $[k . . l]$ for the interval of integers $\{i \in \mathbb{N} \mid k \leq i \leq l\}$.

Given a set $X$ and $n \in \mathbb{N}, X^{n}$ is the set of $n$-dim vectors with values in $X$. For any index $i \in[1 . . n]$, we denote by $\mathbf{v}(i)$ the $i^{t h}$ component of a $n$-dim vector $\mathbf{v}$. We write $\mathbf{0}$ the vector such that $\mathbf{0}(i)=0$ for all $i \in[1 . . n]$. The classical order on $\mathbb{Z}^{n}$ is also denoted $\leq$ and is defined by $\mathbf{v} \leq \mathbf{w}$ if and only if for all $i \in[1 . . n]$, we have $\mathbf{v}(i) \leq \mathbf{w}(i)$. We also define the operation + over $n$-dim vectors of integers in the classical way (ie for $\mathbf{v}$, $\mathbf{v}^{\prime} \in \mathbb{Z}^{n}, \mathbf{v}+\mathbf{v}^{\prime}$ is defined by $\left(\mathbf{v}+\mathbf{v}^{\prime}\right)(i)=\mathbf{v}(i)+\mathbf{v}^{\prime}(i)$ for all $\left.i \in[1 . . n]\right)$.

Let $n \in \mathbb{N}$. A subset $S \subseteq \mathbb{N}^{n}$ is linear if there exist $k+1$ vectors $\mathbf{v}_{0}, \mathbf{v}_{1}, \ldots, \mathbf{v}_{k}$ in $\mathbb{N}^{n}$ such that $S=\left\{\mathbf{v} \mid \mathbf{v}=\overline{\mathbf{v}}_{0}+\lambda_{1} \cdot \mathbf{v}_{1}+\ldots+\lambda_{k} \cdot \mathbf{v}_{k}\right.$ with $\lambda_{i} \in \mathbb{N}$ for all $\left.i \in[1 . . k]\right\}$. A semi-linear set is any finite union of linear sets. We extend the notion of semi-linearity to subsets of $Q \times \mathbb{N}^{n}$ where $Q$ is a finite (non-empty) set.

For an alphabet $\Sigma$, we denote by $\Sigma^{*}$ the set of finite words over $\Sigma$ and $\epsilon$ represents the empty word.

\subsection{Counter machines}

A Minsky machine is a finite control state automaton which manipulates integer variables, called counters. From each control state, the machine can do the following operations : 1) Increment a counter and go to another control state, 2) Test the value of 
a counter, if it is 0 , it passes to a control state, and if not, it decrements the counter and goes to another control state. There is also a control state called the final state (or halting state) from which the machine cannot do anything. The Minsky machine is said to halt when it reaches this control state. We define here a slight extension of Minsky machines.

We call a $n$-dim guarded translation (shortly a translation) any function $t: \mathbb{N}^{n} \rightarrow$ $\mathbb{N}^{n}$ such that there exist $\# \in\{=, \leq\}^{n}, \mu \in \mathbb{N}^{n}$ and $\delta \in \mathbb{Z}^{n}$ with $0 \leq \mu+\delta$ and $\operatorname{dom}(t)=\left\{\mathbf{v} \in \mathbb{N}^{n} \mid \mu \# \mathbf{v}\right\}$ and for all $\mathbf{v} \in \operatorname{dom}(t), t(\mathbf{v})=\mathbf{v}+\delta$. We will sometimes use the encoding $(\#, \mu, \delta)$ to represent a translation. In the following, $T_{n}$ will denote the set of the $n$-dim guarded translations. Let $t=(\#, \mu, \delta)$ be a guarded translation in $T_{n}$. We define the vector $D_{t} \in \mathbb{Z}^{n}$ as follows, $\forall i \in[1 . . n], D_{t}(i)=\delta(i)$. We extend this definition to words of guarded translations, recursively as follows, if $\sigma \in T_{n}^{*}$ and $t \in T_{n}$, we have $D_{t \sigma}=D_{t}+D_{\sigma}$ and by convention, $D_{\epsilon}=\mathbf{0}$.

Definition 1. A $n$-dim counter machine (shortly counter machine) is a finite valuated graph $S=\langle Q, E\rangle$ where $Q$ is a finite set of control states and $E$ is a finite relation $E \subseteq Q \times T_{n} \times Q$

The semantics of a counter machine $S=\langle Q, E\rangle$ is given by its associated transition system $T S(S)=\left\langle Q \times \mathbb{N}^{n}, \rightarrow\right\rangle$ where $\rightarrow \subseteq Q \times \mathbb{N}^{n} \times T_{n} \times Q \times \mathbb{N}^{n}$ is a relation defined as follows :

$$
(q, \mathbf{v}) \stackrel{t}{\rightarrow}\left(q^{\prime}, \mathbf{v}^{\prime}\right) \text { iff } \exists\left(q, t, q^{\prime}\right) \in E \text { such that } \mathbf{v} \in \operatorname{dom}(t) \text { and } \mathbf{v}^{\prime}=t(\mathbf{v})
$$

We write $(q, \mathbf{v}) \rightarrow\left(q^{\prime}, \mathbf{v}^{\prime}\right)$ if there exists $t \in T_{n}$ such that $(q, \mathbf{v}) \stackrel{t}{\rightarrow}\left(q^{\prime}, \mathbf{v}^{\prime}\right)$. The relation $\rightarrow^{*}$ represents the reflexive and transitive closure of $\rightarrow$. Given a configuration $(q, \mathbf{v})$ of $T S(S), \operatorname{Reach}(S,(q, \mathbf{v}))=\left\{\left(q^{\prime}, \mathbf{v}^{\prime}\right) \mid(q, \mathbf{v}) \rightarrow^{*}\left(q^{\prime}, \mathbf{v}^{\prime}\right)\right\}$. Furthermore, we extend the relation $\rightarrow$ to words in $T_{n}^{*}$. We have then $(q, \mathbf{v}) \stackrel{\epsilon}{\rightarrow}(q, \mathbf{v})$ and if $t \in T_{n}$ and $\sigma \in T_{n}^{*}$, $(q, \mathbf{v}) \stackrel{t \sigma}{\rightarrow}\left(q^{\prime \prime}, \mathbf{v}^{\prime \prime}\right)$ if $(q, \mathbf{v}) \stackrel{t}{\rightarrow}\left(q^{\prime}, \mathbf{v}^{\prime}\right) \stackrel{\sigma}{\rightarrow}\left(q^{\prime \prime}, \mathbf{v}^{\prime \prime}\right)$.

Given a counter machine $S=\langle Q, E\rangle$ and an initial configuration $c \in Q \times \mathbb{N}^{n}$, the pair $(S, c)$ is an intialized counter machine. Since, the notations are explicit, in the following we shall write counter machine for both $(S, c)$ and $S$.

It is true that any counter machine can be easily encoded into a Minsky machine. For instance to encode a test of the form $x_{i}=c$, the Minsky machine can decrement $c$ times the counter, test to 0 and increment again $c$ times the counter. Note that this encoding modifies the number of alternations between increasing and decreasing mode for the counters, which is the factor we are interested in when considering reversalboundedness. That is the reason why we propose this extension of Minsky machine. We do not go further for instance extending the guards, because in [11], it is proved that the reachability problem for reversal-bounded counter machines with linear guards (of the form $x=y$ where $x, y$ are two counters variables) is undecidable.

\section{New reversal-bounded counter machines}

\subsection{Reversal-bounded counter machines}

We would like to extend the notion of reversal-bounded to capture and verify a larger class of counter machines. In fact, if we consider the counter machine represented by 


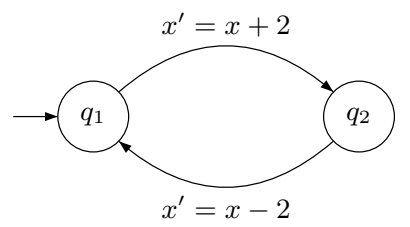

Fig. 1. A simple not reversal-bounded counter machine

the figure 1 with the initial configuration $\left(q_{1}, 0\right)$. Its reachability set is finite equal to $\left\{\left(q_{1}, 0\right),\left(q_{2}, 2\right)\right\}$ and consequently semi-linear but the counter machine is not reversalbounded. We propose here an extension of the notion of reversal-bounded, which allows us to handle such cases and more generally every bounded counter machines.

Given an integer $b \in \mathbb{N}$, we now consider the number of alternations between increasing and decreasing mode when the value of a counter goes above the bound $b$. Let $S=\langle Q, E\rangle$ be a $n$-dim counter machine and $T S(S)=\left\langle Q \times \mathbb{N}^{n}, \rightarrow\right\rangle$. From it, we define another transition system $T S_{b}(S)=\left\langle Q \times\{\downarrow, \uparrow\}^{n} \times \mathbb{N}^{n} \times \mathbb{N}^{n}, \rightarrow_{b}\right\rangle$. Intuitively for a configuration $(q, \mathbf{m}, \mathbf{v}, \mathbf{r}) \in Q \times\{\downarrow, \uparrow\}^{n} \times \mathbb{N}^{n} \times \mathbb{N}^{n}$, the vector $\mathbf{m}$ is used to store the current mode of each counter -increasing $(\uparrow)$ or decreasing $(\downarrow)$-, the vector $\mathbf{v}$ contains the values and the vector $\mathbf{r}$ the numbers of alternations performed over $b$. Formally, we have $(q, \mathbf{m}, \mathbf{v}, \mathbf{r}) \stackrel{t}{\rightarrow} b\left(q^{\prime}, \mathbf{m}^{\prime}, \mathbf{v}^{\prime}, \mathbf{r}^{\prime}\right)$ if and only if the following conditions hold :

1. $(q, \mathbf{v}) \stackrel{t}{\rightarrow}\left(q^{\prime}, \mathbf{v}^{\prime}\right)$

2. for each $i \in[1 . . n]$, the relation expresses by the following array is verified :

\begin{tabular}{|c|c|c|c|c|}
\hline $\mathbf{v}(i)-\mathbf{v}^{\prime}(i)$ & $\mathbf{m}(i)$ & $\mathbf{m}^{\prime}(i)$ & $\mathbf{v}(i)$ & $\mathbf{r}(i)$ \\
\hline \hline$>0$ & $\downarrow$ & $\downarrow$ & - & $\mathbf{r}(i)$ \\
\hline$>0$ & $\uparrow$ & $\downarrow$ & $\leq b$ & $\mathbf{r}(i)$ \\
\hline$>0$ & $\uparrow$ & $\downarrow$ & $>b$ & $\mathbf{r}(i)+1$ \\
\hline$<0$ & $\uparrow$ & $\uparrow$ & - & $\mathbf{r}(i)$ \\
\hline$<0$ & $\downarrow$ & $\uparrow$ & $\leq b$ & $\mathbf{r}(i)$ \\
\hline$<0$ & $\downarrow$ & $\uparrow$ & $>b$ & $\mathbf{r}(i)+1$ \\
\hline$=0$ & $\downarrow$ & $\downarrow$ & - & $\mathbf{r}(i)$ \\
\hline$=0$ & $\uparrow$ & $\uparrow$ & - & $\mathbf{r}(i)$ \\
\hline
\end{tabular}

We denote by $\rightarrow_{b}^{*}$ the reflexive and transitive closure of $\rightarrow_{b}$. Given a configuration $(q, \mathbf{m}, \mathbf{v}, \mathbf{r})$ of $T S_{b}(S), \operatorname{Reach}_{b}(S,(q, \mathbf{m}, \mathbf{v}, \mathbf{r}))=\left\{\left(q^{\prime}, \mathbf{m}^{\prime}, \mathbf{v}^{\prime}, \mathbf{r}^{\prime}\right) \mid(q, \mathbf{m}, \mathbf{v}, \mathbf{r},) \rightarrow_{b}^{*}\right.$ $\left.\left(q^{\prime}, \mathbf{m}^{\prime}, \mathbf{v}^{\prime}, \mathbf{r}^{\prime}\right)\right\}$. We extend this last notation to the configurations of $T S(S)$, saying that if $(q, \mathbf{v}) \in Q \times \mathbb{N}^{n}$ is a configuration of $T S(S)$, then $\operatorname{Reach}_{b}(S,(q, \mathbf{v}))$ is equal to the set $\operatorname{Reach}_{b}(S,(q, \uparrow, \mathbf{v}, \mathbf{0}))$ where $\uparrow$ denotes here the vector with all components equal to $\uparrow$.

Definition 2. Let $b, k \in \mathbb{N}$. A counter machine $(S, c)$ is $k$-reversal-b-bounded if and only if for all $(q, \boldsymbol{m}, \boldsymbol{v}, \boldsymbol{r}) \in \operatorname{Reach}_{b}(S, c)$ and for all $i \in[1 . . n]$, we have $\boldsymbol{r}(i) \leq k$.

We then say that :

1. A counter machine is reversal-bounded if there exist $k, b \in \mathbb{N}$ such that it is $k$ reversal-b-bounded, 
2. For a given $k \in \mathbb{N}$, a counter machine is $k$-reversal-bounded, if there exists $b \in \mathbb{N}$ such that it is $k$-reversal-b-bounded,

3. For a given $b \in \mathbb{N}$, a counter machine is reversal-b-bounded, if there exists $k \in \mathbb{N}$ such that it is $k$-reversal-b-bounded.

We remark that this definition includes the definition of reversal-bounded given in [10], which corresponds to reversal-0-bounded. In comparison to what is presented in [10], there is a slight difference because we do not have here accepting states and consequently we consider all the possible runs of the counter machine as accepted runs. We will see in section 4 that this difference can change some decidability results. Note that in later works [11], the counter machines are also defined without any accepting state.

\subsection{Reachability set}

In [10], it has been proved that the reversal-0-bounded counter machines have an effectively computable semi-linear reachability set. We extend here this result to all the reversal-bounded counter machines.

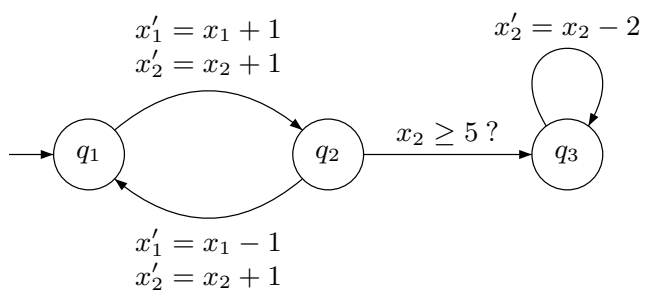

Fig. 2. A 1-reversal-1-bounded counter machine

The idea consists in building from a $k$-reversal-b-bounded counter machine $(S, c)$ a $k$ reversal-0-bounded counter machine $\left(S^{\prime}, c^{\prime}\right)$ as it is done for the counter machine of the figure 2 (with the initial configuration $\left(q_{1},(0,0)\right)$ ) from which we obtain the counter machine represented in the figure 3 (with the initial configuration $\left(\left(q_{1}, 0,0\right),(0,0)\right)$ ). We assume $S=\langle Q, E\rangle$ and $S^{\prime}=\left\langle Q^{\prime}, E^{\prime}\right\rangle$. First we introduce two symbols $\perp$ and $\omega_{b}$ which are not integers. $\omega_{b}$ represents a counter value strictly greater than $b$ and $\perp$ a counter value for which it is not known whether it is greater or not than $b$. The location set $Q^{\prime}$ is then equal to $Q \times B^{n}$ where $B=\{0, \ldots, b\} \cup\left\{\omega_{b}, \perp\right\}$. Intuitively, the counter machine $S^{\prime}$ encodes the run of $S$ and when a counter value in $S$ is under the bound $b$, its value is stored into the control state of $S^{\prime}$ and the corresponding value of the counter in $S^{\prime}$ is 0 , but when the value goes above $b$ in $S$ then it is restored in the counter in $S^{\prime}$. Furthermore $\left(S^{\prime}, c^{\prime}\right)$ being $k$-reversal-0-bounded, we use the results of [10] to compute the reachability set $\operatorname{Reach}\left(S^{\prime}, c^{\prime}\right)$ from which we deduce $\operatorname{Reach}(S, c)$.

Theorem 3. Given a reversal-bounded counter machine, its reachability set is an effectively computable semi-linear set. 


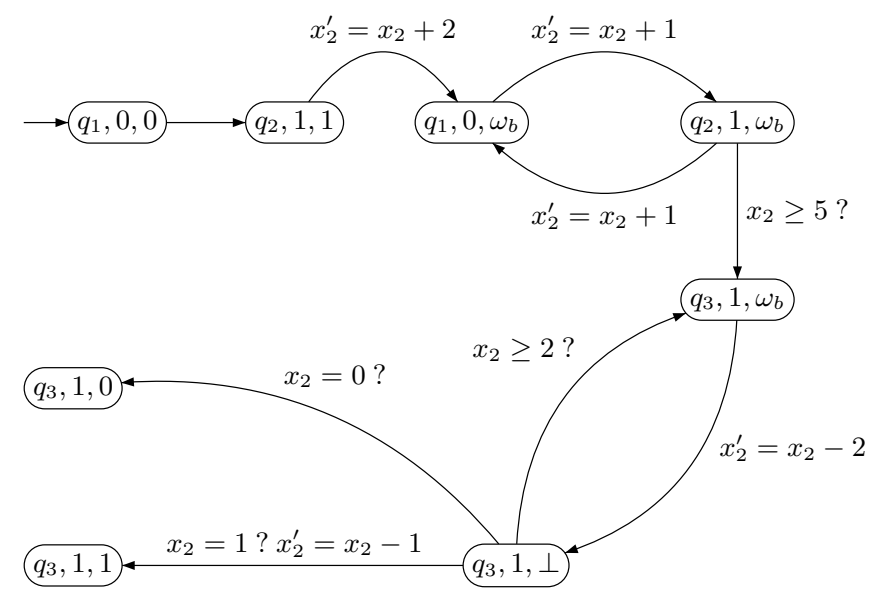

Fig. 3. A 1-reversal-0-bounded counter machine obtained from the counter machine of Fig. 2

\section{Deciding reversal-boundedness}

In this section, we will study the decidabilty of reversal-boundedness.

\subsection{Undecidability}

In [10], the author shows that it is not possible to decide whether a counter machine is reversal-0-bounded or not. We prove here that this theorem is still true when considering reversal-boundedness.

Theorem 4. Verifying if a counter machine is reversal-bounded is undecidable.

Proof. We reduce the halting problem for 2-counters deterministic Minsky Machines. We consider a deterministic Minsky Machine $S$ with the initial configuration $\left(q_{0},(0,0)\right)$ working over two counter variables $x_{1}$ and $x_{2}$. "Deterministic" here means that there is a unique possible run starting on $\left(q_{0},(0,0)\right)$. From $S$, we build a counter machine $S^{\prime}$ working over three counter variables $x_{1}, x_{2}$ and $x_{3}$, such that for each $\left(q, t, q^{\prime}\right) \in E$, we add two control states $q_{1}$ and $q_{2}$ and the transitions $\left(q, t_{1}, q_{1}\right),\left(q_{1}, t_{2}, q_{2}\right)$ and $\left(q_{2}, t, q^{\prime}\right)$ where $t_{1}$ and $t_{2}$ only change the counter variable $x_{3}$ doing $x_{3}^{\prime}=x_{3}+2$ for $t_{1}$ and $x_{3}^{\prime}=x_{3}-1$ for $t_{2}$. Note that $S^{\prime}$ starting on $\left(q_{0},(0,0,0)\right)$ is also deterministic. Furthermore $\left(S^{\prime},\left(q_{0},(0,0,0)\right)\right)$ is reversal-bounded if and only if its unique run is finite, which is equivalent to halting. Since $S^{\prime}$ starting with $\left(q_{0},(0,0,0)\right)$ halts if and only if $S$ starting from $\left(q_{0},(0,0)\right)$ halts and since this last problem is undecidable, we conclude the theorem.

\subsection{Fixing one parameter}

We will see here that fixing one of the parameters is not enough to obtain decidability for the reversal-boundedness. 
Theorem 5. Given $b \in \mathbb{N}$, verifying if a counter machine is reversal-b-bounded is undecidable.

Sketch of Proof, For each $b$ in $\mathbb{N}$, we can reuse the same proof as for the theorem 4 , we can show that the 3 -counter machine $\left(S^{\prime},\left(q_{0},(0,0,0)\right)\right)$ is reversal-b-bounded if and only if the deterministic Minsky machine $(S, c)$ from which it is built halts.

Theorem 6. Given $k \in \mathbb{N}$, verifying if a counter machine is $k$-reversal-bounded is undecidable.

Sketch of Proof. To prove this result we again use the 3-counter machine $S^{\prime}$ with the initial configuration $\left(q_{0},(0,0,0)\right)$ that we complete so that each run can begin with doing at least $k$ alternations between increasing and decreasing mode over any bound. $\square$

\subsection{Fixing the two parameters}

We will now prove that if the two parameters $b$ and $k$ are fixed, it is possible to decide if a counter machine is $k$-reversal-b-bounded. Let $b, k \in \mathbb{N}$ and $(S, c)$ be a counter machine. The idea consists in building a counter machine $\left(S^{\prime}, c^{\prime}\right)$ which will be $(k+1)$-reversal$b$-bounded and which will reach a special control state $q_{e r r}$ if and only if $(S, c)$ is not $k$ reversal-b-bounded. Note that since $\left(S^{\prime}, c^{\prime}\right)$ is reversal-bounded, it is possible to decide whether the control state $q_{e r r}$ is reachable or not. In the control state of $\left(S^{\prime}, c^{\prime}\right)$, we store the mode -increasing $(\uparrow)$ or decreasing $(\downarrow)$ - for each counter and also the number of alternations already performed over $b$. We also add some control states to test at each step if each counter value is strictly greater (denoted by $b_{>}$) or smaller than $b$ (denoted by $b_{\leq}$). The figure 4 gives an example of the counter machine we build to decide if the counter machine from figure 1 with the initial configuration $\left(q_{1}, 0\right)$ is 1-reversal-1bounded.

Theorem 7. Given $b, k \in \mathbb{N}$, verifying if a counter machine is $k$-reversal-b-bounded is decidable.

This result contrasts with the one given in [10], which says that given $k \in \mathbb{N}$, verifying if a counter machine is $k$-reversal-0-bounded is undecidable. This is due to the fact that in [10], the considered counter machines have accepting control states, whereas our definition is equivalent to have all the control states as accepting. In fact, when we define the reversal-bounded counter machines, we consider all the possible runs and not only the one ending in an accepting state.

\subsection{Computing the parameters}

When a counter machine is reversal-bounded, it could be useful to characterize the pairs $(k, b)$ for which it is $k$-reversal-b-bounded, first because it gives us information on the behavior of the counter machine but also because these parameters are involved in the way the reachability set is built as one can see in the proof of theorem 3 and in [10]. 


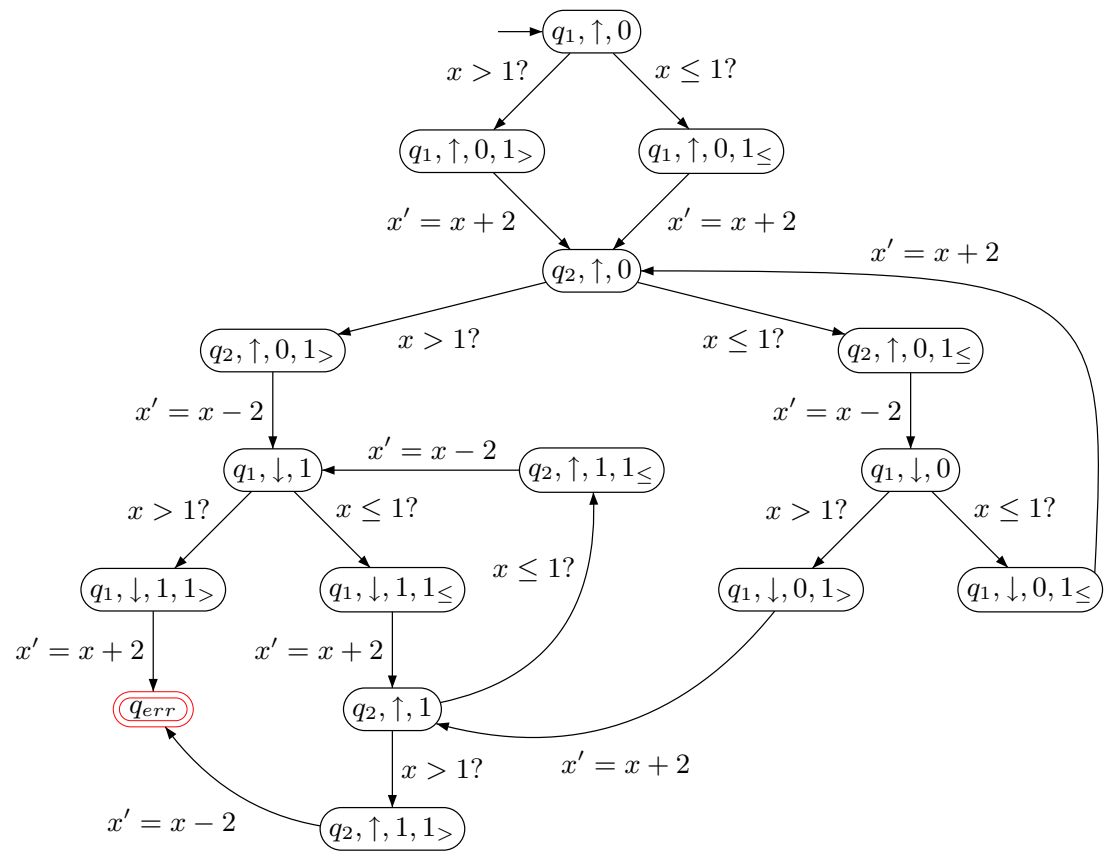

Fig. 4. A 2-reversal-1-bounded counter machine to decide if the counter machine of Fig. 2 is 1-reversal-1-bounded

Let $(S, c)$ be a counter machine. We define the following set to talk about the parameters of reversal-bounded counter machines :

$$
R B(S, c)=\{(k, b) \in \mathbb{N} \times \mathbb{N} \mid(S, c) \text { is } k \text {-reversal-b-bounded }\}
$$

Then $R B(S, c)=\emptyset$ if and only if $(S, c)$ is not reversal-bounded, hence the nonemptiness problem for $R B(S, c)$ is in general not decidable, but this set is recursive (cf. theorem 7). Furthermore, if there exist $(k, b)$ in $R B(S, c)$ and $\left(k^{\prime}, b^{\prime}\right) \in \mathbb{N} \times \mathbb{N}$ such that $(k, b) \leq\left(k^{\prime}, b^{\prime}\right)$ then we know, by definition of reversal-boundedness that $(S, c)$ is also $k^{\prime}$-reversal- $b^{\prime}$-bounded, ie $\left(k^{\prime}, b^{\prime}\right) \in R B(S, c)$. Since the order relation $\leq$ on $\mathbb{N} \times \mathbb{N}$ is a well-ordering we can deduce :

Lemma 8. Let $(S, c)$ be a reversal-bounded counter machine. The set $R B(S, c)$ is upward-closed, it has a finite number of minimal elements, which can effectively be computed.

Sketch of proof.The facts that $R B(S, c)$ is upward closed is a direct consequence of reversal-boundedness. And since $(\mathbb{N} \times \mathbb{N}, \leq)$ is a well-ordering, each of its upwardclosed set has a finite number of minimal elements [8]. To compute the minimal elements, we add "reversal-bounded" counters either to count the number of alternations between increasing and decreasing mode over a bound $b$ or to store the value of a counter each time it changes mode over a given $b$. $\square$ 


\section{Analysis of VASS}

In this section, we recall the definition of Vector Addition System with States and show that the notion of reversal-boundedness we newly introduce is well-suited for the verification of these systems.

\subsection{VASS and their coverability graphs}

Definition 9. A n-dim counter machine $\langle Q, E\rangle$ is a Vector Addition System with States (shortly VASS) if and only if for all transitions $\left(q, t, q^{\prime}\right) \in E, t$ is a guarded translation $(\#, \mu, \delta)$ such that $\#=(\leq, \ldots, \leq)$,

Hence in VASS, it is not possible to test if a counter value is equal to a constant but only if it is greater than a constant.

In [13], the authors provide an algorithm to build from a VASS a labeled tree, the Karp and Miller tree (the algorithm is provided in [7]). The main idea of this construction is to cover in a finite way the reachable configurations using the symbol $\omega$, when a counter is not bounded. They have shown that their algorithm always terminates and that it enjoys some good properties. In particular, this tree can be used to decide the boundedness of a VASS. In [21], the authors have proposed a further construction based on the Karp and Miller tree in order to test the regularity of the language of the unlabeled traces of a VASS. This last construction is known as the coverability graph. To obtain it, the nodes of the Karp and Miller tree with the same labels are grouped together. Formally if $(S, c)$ is a $n$-dim initialized VASS, we denote by $C G(S, c)$ its coverability graph defined as follows, $C G(S, c)=\langle N, \Delta\rangle$ where :

- $N \subseteq Q \times \mathbb{N}_{\omega}^{n}$ is a finite set of nodes,

- $\Delta \subseteq N \times T_{n} \times N$ is a finite set of edges labeled with guarded transitions.

We call a circuit in the coverability graph a path ending in the starting node and a circuit will be said to be elementary if all nodes are different with the exception of the starting and ending nodes. For a vector $\mathbf{w} \in \mathbb{N}_{\omega}^{n}$, we denote by $\operatorname{In} f(\mathbf{w})$ the set $\{i \in[1 . . n] \mid \mathbf{w}(i)=\omega\}$ and $\operatorname{Fin}(\mathbf{w})=[1 . . n] \backslash \operatorname{Inf}(\mathbf{w})$. Using these notions, it has been proved that the coverability graph verifies the following properties.

Let $(S, c)$ be a $n$-dim initialized VASS with $S=\langle Q, E\rangle, T S(S)=\left\langle Q \times \mathbb{N}^{n}, \rightarrow\right\rangle$ its associated transition system and $G=\langle N, \Delta\rangle$ its coverability graph.

Theorem 10. [13,21]

1. If $(q, \boldsymbol{w})$ is a node in $G$, then for all $k \in \mathbb{N}$, there exists $(q, \boldsymbol{v}) \in \operatorname{Reach}(S, c)$ such that for all $i \in \operatorname{In} f(\boldsymbol{w}), k \leq \boldsymbol{v}(i)$ and for all $i \in F$ in $(\boldsymbol{w}), \boldsymbol{w}(i)=\boldsymbol{v}(i)$.

2. For $\sigma \in T_{n}^{*}$, if $c \stackrel{\sigma}{\rightarrow}(q, v)$ then there is a unique path in $G$ labeled by $\sigma$ and leading from c to a node $(q, \boldsymbol{w})$ and for all $i \in F$ in $(\boldsymbol{w}), \boldsymbol{v}(i)=\boldsymbol{w}(i)$.

3. If $\sigma \in T_{n}^{*}$ is a word labeling a circuit in $G$ and $(q, w)$ is the initial node of this circuit, then there exist $(q, v) \in \operatorname{Reach}(S, c)$ and $\left(q^{\prime}, v^{\prime}\right)$ such that $(q, v) \stackrel{\sigma}{\rightarrow}\left(q, v^{\prime}\right)$ and for all $i \in F$ in $(\boldsymbol{w}), \boldsymbol{w}(i)=\boldsymbol{v}(i)=\boldsymbol{v}^{\prime}(i)$. 
From this theorem, we deduce the following lemma, we will then use to decide the reversal-boundedness of a VASS :

Lemma 11. If there exists an elementary circuit $\left(\left(q_{1}, \boldsymbol{w}_{1}\right) \stackrel{t_{1}}{\rightarrow}\left(q_{2}, \boldsymbol{w}_{2}\right) \stackrel{t_{2}}{\rightarrow} \ldots \stackrel{t_{f}}{\rightarrow}\right.$ $\left.\left(q_{1}, \boldsymbol{w}_{1}\right)\right)$ in $G$, then for all $k, l \in \mathbb{N}$, there exist $\boldsymbol{v}_{1}, \ldots, \boldsymbol{v}_{l} \in \mathbb{N}^{n}$ such that :

(i) $c \rightarrow^{*}\left(q_{1}, \boldsymbol{v}_{1}\right) \stackrel{\sigma}{\rightarrow}\left(q_{1}, \boldsymbol{v}_{2}\right) \stackrel{\sigma}{\rightarrow} \ldots \stackrel{\sigma}{\rightarrow}\left(q_{1}, \boldsymbol{v}_{l}\right)$ in $T S(S)$ with $\sigma=t_{1} \ldots t_{f}$, and,

(ii) for all $j \in[1 . . l]$, for all $i \in \operatorname{Inf}\left(\boldsymbol{w}_{1}\right), k \leq \boldsymbol{v}_{j}(i)$ and for all $i \in$ Fin $\left(\boldsymbol{w}_{1}\right)$, $\boldsymbol{w}_{1}(i)=\boldsymbol{v}_{j}(i)$.

\subsection{Deciding if a VASS is reversal- $b$-bounded}

In this section, we show that its possible to decide if a VASS is reversal- $b$-bounded using a characterization over its coverability graph.

Let $S=\langle Q, E\rangle$ be a $n$-dim counter machine. We build a $2 n$-dim counter machine $\widetilde{S}=\left\langle Q^{\prime}, E^{\prime}\right\rangle$ adding for each counter another counter, whose role is to count the alternation of the first counter between increasing and decreasing mode. Formally, $Q^{\prime}=Q \times\{\uparrow, \downarrow\}^{n}$ and $T^{\prime}$ is built as follows, for each $\left(q,(\#, \mu, \delta), q^{\prime}\right) \in E$ and $\mathbf{m}, \mathbf{m}^{\prime} \in\{\uparrow, \downarrow\}^{n}$, we have $\left((q, \mathbf{m}),\left(\#^{\prime}, \mu^{\prime}, \delta^{\prime}\right),\left(q^{\prime}, \mathbf{m}^{\prime}\right)\right) \in E^{\prime}$ if and only if :

- for all $i \in[1 . . n], \#^{\prime}(i)=\#(i), \mu^{\prime}(i)=\mu_{i}$ and $\delta^{\prime}(i)=\delta(i)$;

- for all $i \in[n+1 . .2 n]$, \# $\#^{\prime}(i) \in\{\leq\}$ and $\mu^{\prime}(i)=0$;

- $\delta, \mathbf{m}, \mathbf{m}^{\prime}$ and $\delta^{\prime}$ satisfy for all $i \in[1 . . n]$ the conditions described in the following array :

\begin{tabular}{|c|c|c|c|}
\hline$\delta(i)$ & $\mathbf{m}(i)$ & $\mathbf{m}^{\prime}(i)$ & $\delta^{\prime}(n+i)$ \\
\hline \hline$=0$ & $\uparrow$ & $\uparrow$ & 0 \\
\hline$=0$ & $\downarrow$ & $\downarrow$ & 0 \\
\hline$>0$ & $\uparrow$ & $\uparrow$ & 0 \\
\hline$>0$ & $\downarrow$ & $\uparrow$ & 1 \\
\hline$<0$ & $\downarrow$ & $\downarrow$ & 0 \\
\hline$<0$ & $\uparrow$ & $\downarrow$ & 1 \\
\hline
\end{tabular}

By construction, we remark that if $S$ is a VASS then $\widetilde{S}$ is a VASS too. We define then the relation $\sim \in\left(Q \times\{\uparrow, \downarrow\}^{n} \times \mathbb{N}^{n} \times \mathbb{N}^{n}\right) \times\left(Q \times\{\uparrow, \downarrow\}^{n} \times \mathbb{N}^{2 n}\right)$ between the configurations of $T S_{0}(S)$ and the ones of $T S(\widetilde{S})$ saying that $(q, \mathbf{m}, \mathbf{v}, \mathbf{r}) \sim\left(q^{\prime}, \mathbf{m}^{\prime}, \mathbf{v}^{\prime}\right)$ if and only if :

$-q=q^{\prime}$

$\mathbf{-} \mathbf{m}=\mathbf{m}^{\prime}$,

- for all $i \in[1 . . n], \mathbf{v}(i)=\mathbf{v}^{\prime}(i)$ and $\mathbf{r}(i)=\mathbf{v}^{\prime}(n+i)$.

The relation $\sim$ is a bisimulation between $T S_{0}(S)$ and $T S(\widetilde{S})$. Given an initial configuration $c=(q, \mathbf{v})$, we have $(q, \uparrow, \mathbf{v}, \mathbf{0}) \sim(q, \uparrow,(\mathbf{v}, \mathbf{0}))$. Hence, if we denote by $\widetilde{c}$ the triple $(q, \uparrow,(\mathbf{v}, \mathbf{0}))$, we can deduce that the VASS $(S, c)$ is reversal-0-bounded if and only if there exists $k \in \mathbb{N}$ such that for all $(q, \mathbf{m}, \mathbf{v}) \in \operatorname{Reach}(\widetilde{S}, \widetilde{c})$, for all $i \in[1 . . n]$, $\mathbf{v}(n+i) \leq k$. Using the coverability graph of $(\widetilde{S}, \widetilde{c})$, this last property is decidable for a VASS. Generalizing this method for any $b \in \mathbb{N}$, counting only the alternations that are done above $b$, we can deduce that :

Theorem 12. Given $b \in \mathbb{N}$, verifying if a VASS is reversal-b-bounded is decidable. 


\subsection{Deciding if a VASS is reversal-bounded}

We will now show that the analysis of the coverabilty graph of $(\widetilde{S}, \widetilde{c})$ allows us to decide if a VASS is reversal-bounded (without a fixed bound). Note that this is not a direct consequence of the previous theorem, because it is not possible to enumerate the different bounds $b$ and test if the VASS is reversal- $b$-bounded, since this method never terminates when the VASS is not reversal-bounded.

Lemma 13. A n-dim VASS $(S, c)$ is reversal-b-bounded if and only if for all $i \in[1 . . n]$, all nodes $(q, \boldsymbol{w})$ belonging to an elementary circuit labeled by $\sigma \in T_{n}^{*}$ of $C G(\widetilde{S}, \widetilde{c})$ with $D_{\sigma}(n+i)>0$ verify $\boldsymbol{w}(i) \leq b$.

In other words, this last lemma states that $(S, c)$ is reversal-b-bounded if and only if for all $i \in[1 . . n]$, there is no elementary circuit in the coverability graph $C G(\widetilde{S}, \widetilde{c})$ which strictly increases the $(n+i)$-th counter and which has a node, whose $i$-th component is strictly greater than $b$ or equal to $\omega$. In fact, applying the lemma 11 , we deduce that if such an elementary circuit exists, we can build a run of the VASS $(S, c)$ which does not respect the definition of reversal- $b$-boundedness. The details of the proof can be found in [7].

For a VASS $(S, c)$, the lemma 13 gives us a necessary and sufficient condition over the coverability graph of $(\widetilde{S}, \widetilde{c})$, and this condition can effectively be tested. This allows us to deduce the following decidability result.

Theorem 14. Verifying if a VASS is reversal-bounded is decidable.

Unfortunately, the decision algorithm we propose here builds entirely the coverability graph of a VASS, and this building is known to be non-primitive-recursive in space (some details can be found in [12]).

\section{Perspectives}

In [4], the authors have proved that some liveness problems are decidable for reversal-0bounded counter machines and others not. For instance, it is decidable to verify if a run of a reversal-bounded counter machine passes infinitely often through a semilinear set of possible configurations; but the same problem becomes undecidable when all the runs are considered. It seems that this result can easily be extended to the class of reversalbounded counter machines, we have introduced. It would then pave the way to verify more complex properties than reachability over reversal-bounded counter machines. It could also be interesting to look at these liveness problems in the particular case of reversal-bounded VASS.

An other perspective for our work would be to use reversal-bounded counter machines to analyze counter machines which are not necessarily reversal-bounded. In fact, we have seen with the proof of theorem 7 , that for any $k, b \in \mathbb{N}$ and from any counter machine, it is possible to build another counter machine, which is $k$-reversal-b-bounded and whose runs represent an under-approximation of the set of runs of the first one. We could consequently build a tool which given a counter machine would build successively, incrementing the parameters $k$ and $b$, the corresponding $k$-reversal-b-bounded 
counter machines, and would test at each step if the reachability set of the initial counter machine has been built (this can be easily done, since this set is a fixpoint of the transition relation). This algorithm might never terminate, if the reachability set is not semilinear for instance, but it will refine at each step the under-approximation of the reachability set.

\section{References}

1. A. Annichini, A. Bouajjani, and M. Sighireanu. TReX: A tool for reachability analysis of complex systems. In CAV'01, volume 2102 of LNCS, pages 368-372. Springer, 2001.

2. T. Araki and T. Kasami. Decidable problems on the strong connectivity of Petri net reachability sets. Theor. Comput. Sci., 4(1):99-119, 1977.

3. S. Bardin, J. Leroux, and G. Point. FAST extended release. In CAV'06, volume 4144 of LNCS, pages 63-66. Springer, 2006.

4. Z. Dang, O. H. Ibarra, and P. San Pietro. Liveness verification of reversal-bounded multicounter machines with a free counter. In FSTTCS'01, volume 2245 of LNCS, pages 132-143. Springer, 2001.

5. J. Esparza. Petri nets, commutative context-free grammars, and basic parallel processes. Fundam. Inform., 31(1):13-25, 1997.

6. A. Finkel and J. Leroux. How to compose Presburger-accelerations: Applications to broadcast protocols. In FSTTCS'02, volume 2556 of LNCS, pages 145-156. Springer, 2002.

7. A. Finkel and A. Sangnier. Reversal-bounded counter machines revisited. Research report, Laboratoire Spécification et Vérification, ENS Cachan, 2008.

8. G. Higman. Ordering by divisibility in abstract algebras. Proc. London Math. Soc. (3), 2:326-336, 1952.

9. J. E. Hopcroft and J.-J. Pansiot. On the reachability problem for 5-dimensional vector addition systems. Theor. Comput. Sci., 8:135-159, 1979.

10. O. H. Ibarra. Reversal-bounded multicounter machines and their decision problems. J. ACM, 25(1):116-133, 1978.

11. O. H. Ibarra, J. Su, Z. Dang, T. Bultan, and R. A. Kemmerer. Counter machines and verification problems. Theor. Comput. Sci., 289(1):165-189, 2002.

12. M. Jantzen. Complexity of place/transition nets. In Advances in Petri Nets, volume 254 of LNCS, pages 413-434. Springer, 1986.

13. R. M. Karp and R. E. Miller. Parallel program schemata: A mathematical model for parallel computation. In FOCS'67, pages 55-61. IEEE, 1967.

14. S. R. Kosaraju. Decidability of reachability in vector addition systems (preliminary version). In STOC'82, pages 267-281. ACM, 1982.

15. L. H. Landweber and E. L. Robertson. Properties of conflict-free and persistent Petri nets. J. ACM, 25(3):352-364, 1978.

16. Homepage of LASH. http://www.montefiore.ulg.ac.be/ boigelot/research/lash .

17. J. Leroux and G. Sutre. Flat counter almost everywhere! In ATVA'05, volume 3707 of LNCS, pages 474-488. Springer, 2005.

18. E. W. Mayr. Persistence of vector replacement systems is decidable. Acta Inf., 15:309-318, 1981.

19. E. W. Mayr. An algorithm for the general Petri net reachability problem. SIAM J. Comput., 13(3):441-460, 1984.

20. M. L. Minsky. Computation: finite and infinite machines. Prentice-Hall, Inc., Upper Saddle River, NJ, USA, 1967.

21. R. Valk and G. Vidal-Naquet. Petri nets and regular languages. J. Comput. Syst. Sci., 23(3):299-325, 1981. 